\title{
ON THE NUMBERS OF 2-WEIGHTS, UNIPOTENT CONJUGACY CLASSES, AND IRREDUCIBLE BRAUER 2-CHARACTERS OF FINITE CLASSICAL GROUPS
}

\author{
JIANBEI AN AND MARSTON CONDER
}

(Communicated by Ronald M. Solomon)

\begin{abstract}
A closed expression for the number of unipotent conjugacy classes of a classical group $G$ is given and Alperin's weight conjecture is confirmed for $G$ globally and for a symplectic or odd-dimensional orthogonal group block by block.
\end{abstract}

\section{INTRODUCTION}

Let $G$ be a classical symplectic or orthogonal group defined over a field of odd characteristic. A 2-block $B$ of $G$ is labelled by a semisimple $2^{\prime}$-element $s^{*}$ in $G$ by results of Cabanes and Enguehard [7] and the first author [3]. Let $\mathscr{W}(B)$ be the number of 2-weights in $G$ associated with $B$ and $\mathscr{U}_{G}\left(s^{*}\right)$ the number of unipotent conjugacy classes of $C_{G}\left(s^{*}\right)$, and let $l(B)$ be the number of irreducible Brauer 2-characters in $B$. In this paper we prove that $\mathscr{W}(B)=\mathscr{U}_{G}\left(s^{*}\right)=l(B)$ for each block $B$ of a symplectic or odd-dimensional orthogonal group $G$. In addition, $\mathscr{W}(B)=\mathscr{U}_{G}\left(s^{*}\right)$ and $\mathscr{U}_{G_{0}}\left(s^{*}\right)=l\left(B^{\prime}\right)$ when $G$ is an even-dimensional orthogonal group, where $G_{0}$ is the special orthogonal group, $\mathscr{U}_{G_{0}}\left(s^{*}\right)$ is the number of unipotent conjugacy classes in $C_{G_{0}}\left(s^{*}\right)$, and $B^{\prime}$ is a block of $G_{0}$ covered by $B$. In the latter case, we could not get the equation $\mathscr{U}_{G}\left(s^{*}\right)=l(B)$ because we do not know how to get $l(B)$. We give as corollaries a closed expression for the number of unipotent conjugacy classes of $G$, and get an affirmative answer for Alperin's weight conjecture globally for $G$ and block by block for a symplectic or odd-dimensional orthogonal group $G$. Notice that the three numbers $\mathscr{W}(B), \mathscr{U}_{G}\left(s^{*}\right)$, and $l(B)$ are also the same for a 2-block $B$ of a general linear or unitary group by results of [1-2], [4], and [10].

In $\S 1$ we use the generating function given by Wall [14] for unipotent conjugacy classes of a symplectic or orthogonal group to show that $\mathscr{W}(B)=\mathscr{U}_{G}\left(s^{*}\right)$, and we give a closed formula for the number of unipotent conjugacy classes. In $\S 2$ we use the results of Broue [5] to show that $\mathscr{U}_{G_{0}}\left(s^{*}\right)=l\left(B^{\prime}\right)$.

Received by the editors August 15, 1993.

1991 Mathematics Subject Classification. Primary 20C20, 20G40.

(C)1995 American Mathematical Society 


\section{THE NUMBERS OF WEIGHTS FOR 2-BLOCKS}

Let $\mathbb{F}_{q}$ be a field of $q$ elements with odd characteristic, and let $V$ be a non-degenerate finite-dimensional symplectic or orthogonal space over $\mathbb{F}_{q}$. In addition, let $I(V)$ be the group of all isometries of $V$ and $I_{0}(V)$ the subgroup of $I(V)$ of isometries of determinant 1 . Thus $I(V)=I_{0}(V)$ if $V$ is symplectic. Let $G=I_{0}(V)$, and let $G^{*}$ be the dual group of $G$. If $s$ is a semisimple element of $G^{*}$, then let $(s)$ be the conjugacy class of $G^{*}$ containing $s$, and let $\mathscr{E}(G,(s))$ be the set of the irreducible constituents of Deligne-Lusztig generalized characters associated with $(s)$ (see [6, p. 57]). If $s$ is a semisimple $2^{\prime}$-element of $G^{*}$, let

$$
\mathscr{E}_{2}(G,(s))=\bigcup_{u} \mathscr{E}(G,(s u)),
$$

where $u$ runs over all 2-elements of $C_{G^{*}}(s)$. By [7, Theorem 13] and [5, Theorem 3.2], $\mathscr{E}_{2}(G,(s))$ is a 2-block, so that a 2-block $B$ of $I(V)$ covers a 2-block $\mathscr{E}_{2}(G,(s))$. By [3, (5B)(c)], $B$ covers another block $\mathscr{E}_{2}\left(G,\left(s^{\prime}\right)\right)$ if and only if $s$ and $s^{\prime}$ are conjugate in $I\left(V^{*}\right)$, where $V^{*}$ is the underlying space of $G^{*}$. For each semisimple $2^{\prime}$-element $s$ in $I_{0}(V)^{*}$ a dual element $s^{*}$ in $I_{0}(V)$ of $s$ is defined by [3, (4A)] and $s^{*}$ is determined uniquely by $s$ up to conjugacy in $I(V)$. We shall say that $s^{*}$ is a semisimple label of $B$. Thus a semisimple label of $B$ is determined uniquely up to conjugacy in $I(V)$. In this section, we shall show that the number of weights for a 2-block of $I(V)$ with semisimple label $s^{*}$ is the number $\mathscr{U}_{I(V)}\left(s^{*}\right)$ of unipotent conjugacy classes of $C_{I(V)}\left(s^{*}\right)$. In particular, we shall get a closed formula for the number of unipotent conjugacy classes of $I(V)$.

First of all, we consider the symplectic group $\operatorname{Sp}(V)$. We shall need the following lemma.

Lemma (1A). The following identity holds:

$$
\prod_{j=1}^{\infty}\left(1-t^{2 j}\right) \prod_{j=1}^{\infty}\left(1+t^{j}\right)=\sum_{i=1}^{\infty} t^{i(i-1)} \text {. }
$$

Proof. If $C(2, m)$ is the number of 2-cores of rank $m$, then

$$
C(2, m)= \begin{cases}1 & \text { if } m=\frac{i(i-1)}{2} \text { for some } i \in \mathbb{N}, \\ 0 & \text { otherwise, }\end{cases}
$$

where $\mathbb{N}$ is the set of all natural numbers. Now let $P(t)$ and $F_{2}(t)$ be the generating functions of partitions and 2-cores, respectively. Then

$$
P(t)=\prod_{i=1}^{\infty} \frac{1}{1-t^{i}} \text { and } F_{2}(t)=\sum_{i=1}^{\infty} t^{\frac{i(i-1)}{2}},
$$

and by [12, Proposition 3.3]

$$
F_{2}(t)=\left(P\left(t^{2}\right)\right)^{-2} P(t),
$$

therefore

$$
F_{2}(t)=\prod_{j=1}^{\infty}\left(1-t^{2 j}\right)^{2} \prod_{i=1}^{\infty} \frac{1}{1-t^{i}}=\prod_{j=1}^{\infty}\left(1-t^{2 j}\right) \prod_{i=1}^{\infty}\left(1+t^{i}\right) .
$$

Thus (1.1) holds. 
As a consequence of (1A) we have the following corollary.

Corollary (1B). Let $\pi_{o}(m)$ and $\pi_{e}(m)$ be the number of odd and even partitions of rank $m$, respectively, where an odd partition is a partition with odd parts and an even partition is a partition with even parts. Then

$$
\pi_{o}(m)=\sum_{j=1}^{m} \pi_{e}(j) C(2, m-j)=\sum_{i=1}^{m} \pi_{e}(m-i) C(2, i),
$$

where $C(2, l)$ is the number of 2-cores of rank $l$ given by (1.2).

Proof. Let $g_{o}(t)$ and $g_{e}(t)$ be the generating functions of odd and even partitions, respectively. Then

$$
g_{o}(t)=\prod_{l=1}^{\infty}\left(1+t^{l}\right) \quad \text { and } \quad g_{e}(t)=\prod_{l=1}^{\infty} \frac{1}{1-t^{2 l}}
$$

By (1A) $g_{o}(t)=g_{e}(t) F_{2}(t)$, and so (1.5) holds.

Proposition (1C). Let $G$ be the symplectic group $\operatorname{Sp}(V)=\operatorname{Sp}(2 n, q)$ and $B_{0}$ the principal 2-block of $G$, and let $\mathscr{W}\left(B_{0}\right)$ be the number of $B_{0}$-weights. Then $\mathscr{W}\left(B_{0}\right)$ is the number $\mathscr{U}_{G}(1)$ of unipotent conjugacy classes of $G$. In particular,

$$
\mathscr{U}_{G}(1)=\sum_{\kappa} f_{X-1, \kappa}
$$

where $\kappa$ runs over all 2-cores with $|\kappa| \leq n$ and $f_{X-1, \kappa}$ is the number of pairs $\left(\lambda_{1}, \lambda_{2}\right)$ of partitions $\lambda_{i}$ such that $\left|\lambda_{1}\right|+\left|\lambda_{2}\right|=n-|\kappa|$.

Proof. Let

$$
f_{X-1}=\sum_{\kappa} f_{X-1, \kappa}
$$

where $\kappa$ runs over all 2-cores with $|\kappa| \leq n$. By [3, (6D)(d)] $\mathscr{W}\left(B_{0}\right)=f_{X-1}$.

Let $k(m, l)$ be the number of $m$-tuples $\left(\lambda_{1}, \lambda_{2}, \ldots, \lambda_{m}\right)$ of partitions $\lambda_{i}$ such that

$$
\sum_{i=1}^{m}\left|\lambda_{i}\right|=l
$$

Then by [13, p. 237],

$$
P(t)^{m}=\sum_{l \geq 0} k(m, l) t^{l}
$$

The generating function of $\mathscr{W}\left(B_{0}\right)$ is

$$
f(t)=\left(\sum_{l=0}^{\infty} k(2, l) t^{2 l}\right) F_{2}\left(t^{2}\right)=\left(\prod_{i=1}^{\infty} \frac{1}{1-t^{2 i}}\right)^{2} F_{2}\left(t^{2}\right),
$$

where $F_{2}(t)$ is the generating function of 2 -cores given by (1.3). By [14, p. 38], the generating function of the number of unipotent conjugacy classes in $G$ is

$$
F_{-}(t)=\prod_{i=1}^{\infty} \frac{\left(1+t^{2 i}\right)^{2}}{1-t^{2 i}}
$$


Now set $x=t^{2}$. It suffices to show that

$$
\prod_{i=1}^{\infty} \frac{\left(1+x^{i}\right)^{2}}{\left(1-x^{i}\right)}=\left(\prod_{i=1}^{\infty} \frac{1}{1-x^{i}}\right)^{2} F_{2}(x) .
$$

By (1A) $F_{2}(x)=\prod_{i=1}^{\infty}\left(1-x^{2 i}\right)\left(1+x^{i}\right)$, so that $F_{2}(x)=\prod_{i=1}^{\infty}\left(1-x^{i}\right)\left(1+x^{i}\right)^{2}$, and (1.8) follows. This completes the proof.

We now consider an orthogonal group $\mathrm{O}(V)$. Let $B_{0}$ be the principal 2block of $\mathrm{O}(V)$ and $\mathscr{W}\left(B_{0}\right)$ the number of $B_{0}$-weights. Then $\mathscr{W}\left(B_{0}\right)$ is given by [3, (6D)]. If $\operatorname{dim} V$ is odd, then in the notation of [3, (6D)], $\mathscr{W}\left(B_{0}\right)$ is the number

$$
f_{X-1}=\sum_{\kappa_{1}, \kappa_{2}, \kappa} f_{X-1, \kappa_{1}, \kappa_{2}, \kappa}
$$

where $\kappa_{1}$ and $\kappa_{2}$ run over all 2-cores such that $\left|\kappa_{1}\right|$ and $\left|\kappa_{2}\right|$ are odd and even, respectively, $\kappa$ runs over all 2-cores and $f_{X-1, \kappa_{1}, \kappa_{2}, \kappa}$ is the number of 4-tuples $\left(\lambda_{1}, \lambda_{2}, \lambda_{3}, \lambda_{4}\right)$ of partitions $\lambda_{i}$ such that

$$
\left|\lambda_{1}\right|+\left|\lambda_{2}\right|+\left|\lambda_{3}\right|+\left|\lambda_{4}\right|=\frac{1}{4}\left(\operatorname{dim} V-\left|\kappa_{1}\right|-\left|\kappa_{2}\right|-2|\kappa|\right) .
$$

Let $D(V)$ be the discriminant of $V$ and $\sigma$ a non-square element of $\mathbb{F}_{q}$. If $\operatorname{dim} V$ is even, then $\mathscr{W}\left(B_{0}\right)$ is also given by (1.9), where $\kappa_{1}$ and $\kappa_{2}$ run over all 2-cores such that $D(V)=\sigma^{\left|\kappa_{1}\right|}$ and $\left|\kappa_{1}\right|,\left|\kappa_{2}\right|$ are either both odd or both even, $\kappa$ runs over all 2-cores and $f_{X-1, \kappa_{1}, \kappa_{2}, \kappa}$ is the number of 4-tuples $\left(\lambda_{1}, \lambda_{2}, \lambda_{3}, \lambda_{4}\right)$ of partitions $\lambda_{i}$ such that

$$
\left|\lambda_{1}\right|+\left|\lambda_{2}\right|+\left|\lambda_{3}\right|+\left|\lambda_{4}\right|=\frac{1}{4}\left(\operatorname{dim} V-\left|\kappa_{1}\right|-\left|\kappa_{2}\right|-2|\kappa|\right) .
$$

If $\operatorname{dim} V=w$ and the type $\eta(V)$ of $V$ is \pm , then we denote by $\mathscr{W}_{w}^{ \pm}$the number $\mathscr{W}\left(B_{0}\right)$. Thus $\mathscr{W}_{w}^{+}=\mathscr{W}_{w}^{-}$if $w$ is odd. Let $g(t)$ be the generating function of $\mathscr{W}_{w}^{+}+\mathscr{W}_{w}^{-}$, so that

$$
g(t)=\left(\sum_{w=1}^{\infty} k(4, w) t^{4 w}\right) F_{2}\left(t^{2}\right) F_{2}(t)^{2} .
$$

By (1.4) and (1.7)

$$
\begin{aligned}
g(t) & =P\left(t^{4}\right)^{4} P\left(t^{4}\right)^{-2} P\left(t^{2}\right) P\left(t^{2}\right)^{-4} P(t)^{2}=P\left(t^{4}\right)^{2} P\left(t^{2}\right)^{-3} P(t)^{2} \\
& =\prod_{k=1}^{\infty} \frac{1+t^{k}}{\left(1-t^{k}\right)\left(1+t^{2 k}\right)^{2}} .
\end{aligned}
$$

On the other hand, if $\mathscr{U}_{w}^{ \pm}$is the number of unipotent conjugacy classes of $\mathrm{O}(V)$ such that $\operatorname{dim} V=w$ and $\eta(V)= \pm$, then by [14, (2.6.17)], the generating function of $\mathscr{U}_{w}^{+}+\mathscr{U}_{w}^{-}$is

$$
F_{+}^{+}(t)=\prod_{k=1}^{\infty} \frac{\left(1+t^{2 k-1}\right)^{2}}{1-t^{2 k}}
$$

But

$$
\prod_{k=1}^{\infty}\left(1+t^{k}\right)^{2}=\prod_{k=1}^{\infty}\left(1+t^{2 k-1}\right)^{2}\left(1+t^{2 k}\right)^{2}
$$


so it follows that $g(t)=F_{+}^{+}(t)$. In particular, if $w$ is odd, then $\mathscr{W}\left(B_{0}\right)=$ $\mathscr{W}_{w}^{+}=\mathscr{W}_{w}^{-}$is the number of unipotent conjugacy classes of $\mathrm{O}(V)$.

Suppose $w=2 n$ for some integer $n$. Then

$$
F_{2}(t)+F_{2}(-t)=\sum_{\kappa} 2 t^{|\kappa|} \quad F_{2}(t)-F_{2}(-t)=\sum_{\kappa^{\prime}} 2 t^{\left|\kappa^{\prime}\right|},
$$

where $\kappa$ and $\kappa^{\prime}$ run over all 2-cores such that $|\kappa|$ and $\left|\kappa^{\prime}\right|$ are even and odd, respectively. Thus the generating function $h(t)$ of $\mathscr{W}_{2 n}^{+}-\mathscr{W}_{2 n}^{-}$is given by

$$
\left(\sum_{j=1}^{\infty} k(4, j) t^{4 j}\right) F_{2}\left(t^{2}\right)\left[\left(\frac{1}{2}\left(F_{2}(t)+F_{2}(-t)\right)\right)^{2}-\left(\frac{1}{2}\left(F_{2}(t)-F_{2}(-t)\right)\right)^{2}\right],
$$

so that $h(t)=P\left(t^{4}\right)^{4} F_{2}\left(t^{2}\right) F_{2}(t) F_{2}(-t)$. By (1.4) and (1.7)

$$
\begin{aligned}
h(t) & =P\left(t^{4}\right)^{4} P\left(t^{4}\right)^{-2} P\left(t^{2}\right) P\left(t^{2}\right)^{-2} P(t) P\left((-t)^{2}\right)^{-2} P(-t) \\
& =P\left(t^{4}\right)^{2} P\left(t^{2}\right)^{-3} P(t) P(-t) \\
& =\prod_{k=1}^{\infty} \frac{1}{\left(1-t^{4 k}\right)^{2}} \prod_{k=1}^{\infty}\left(1-t^{2 k}\right)^{3} \prod_{k=1}^{\infty} \frac{1}{\left(1-t^{k}\right)\left(1-(-t)^{k}\right)} \\
& =\prod_{k=1}^{\infty} \frac{1}{\left(1-t^{4 k}\right)^{2}} \prod_{k=1}^{\infty}\left(1-t^{2 k}\right)^{3} \prod_{k=1}^{\infty} \frac{1}{\left(1-t^{2 k}\right)^{2}} \prod_{k=1}^{\infty} \frac{1}{\left(1-t^{4 k-2}\right)} \\
& =\prod_{k=1}^{\infty} \frac{1}{\left(1-t^{4 k}\right)^{2}} \prod_{k=1}^{\infty}\left(1-t^{2 k}\right) \prod_{k=1}^{\infty} \frac{1}{\left(1-t^{4 k-2}\right)} .
\end{aligned}
$$

But $\prod_{k=1}^{\infty}\left(1-t^{4 k-2}\right)^{-1}=\prod_{k=1}^{\infty}\left(1+t^{2 k}\right)$ (cf. $[14$, p. 42]), so

$$
h(t)=\prod_{k=1}^{\infty} \frac{1}{\left(1-t^{4 k}\right)^{2}} \prod_{k=1}^{\infty}\left(1-t^{4 k}\right)=P\left(t^{4}\right) .
$$

By $[14,(2.6 .18)] P\left(t^{4}\right)$ is the generating function $F_{+}^{-}(t)$ of $\mathscr{U}_{2 n}^{+}-\mathscr{U}_{2 n}^{-}$. Thus $h(t)=F_{+}^{-}(t)$ and then $\mathscr{W}\left(B_{0}\right)$ is the number of unipotent conjugacy classes of $\mathrm{O}(V)$. So we have proved the following proposition.

Proposition (1D). Let $B_{0}$ be the principal 2-block of $\mathrm{O}(V)$, and let $\mathscr{W}\left(B_{0}\right)$ be the number of $B_{0}$-weights. Then $\mathscr{W}\left(B_{0}\right)$ is the number $\mathscr{U}_{\mathrm{O}(V)}(1)$ of unipotent conjugacy classes of $\mathrm{O}(V)$. In particular, if $D(V)$ is the discriminant of $V$ and $\sigma$ is a non-square element of $\mathbb{F}_{q}$, then

$$
\mathscr{U}_{\mathrm{O}(V)}(1)=\sum_{\kappa_{1}, \kappa_{2}, \kappa} f_{X-1, \kappa_{1}, \kappa_{2}, \kappa},
$$

where $\kappa_{1}, \kappa_{2}, \kappa$ run over all 2-cores such that $D(V)=\sigma^{\left|\kappa_{1}\right|}$ and $f_{X-1, \kappa_{1}, \kappa_{2}, \kappa}$ is the number of 4-tuples $\left(\lambda_{1}, \lambda_{2}, \lambda_{3}, \lambda_{4}\right)$ of partitions $\lambda_{i}$ such that

$$
\left|\lambda_{1}\right|+\left|\lambda_{2}\right|+\left|\lambda_{3}\right|+\left|\lambda_{4}\right|=\frac{1}{4}\left(\operatorname{dim} V-\left|\kappa_{1}\right|-\left|\kappa_{2}\right|-2|\kappa|\right) .
$$

Now let $B$ be a 2-block of $I(V)$ covering $\mathscr{E}_{2}\left(I_{0}(V),(s)\right)$ for some semisimple $2^{\prime}$-element $s$ of $I_{0}(V)^{*}$, and let $s=\prod_{\Gamma} s_{\Gamma}$ be the primary decomposition of $s$ in $G^{*}$ in the sense of $\left[11\right.$, p. 125]. In addition, let $m_{\Gamma}(s)$ be the multiplicity of $\Gamma$ in $s$, let $V_{X-1}^{*}$ be the underlying space of $s_{X-1}$, and let $V_{X-1}$ be the 
space dual of $V_{X-1}^{*}$ in the sense of $[11,(3.1)]$. By [3, (6E)] the number $\mathscr{W}(B)$ of $B$-weights is $\prod_{\Gamma} f_{\Gamma}$, where $f_{\Gamma}$ is the number of partitions of rank $m_{\Gamma}(s)$ except when $\Gamma=X-1$, in which case $f_{X-1}$ is given by (1.6) or (1.9) with $\operatorname{dim} V$ replaced by $\operatorname{dim} V_{X-1}$ according as $V$ is symplectic or orthogonal. Thus $f_{X-1}$ is the number of unipotent conjugacy classes of $I\left(V_{X-1}\right)$ by (1C) and (1D). By $[11,(1.13)]$

$$
C_{I_{0}(V)^{*}}(s)^{*} \simeq \prod_{\Gamma \neq X-1} \mathrm{GL}\left(m_{\Gamma}(s), \varepsilon_{\Gamma} q^{\delta_{\Gamma}}\right) \times I_{0}\left(V_{X-1}\right),
$$

where $\varepsilon_{\Gamma}$ and $\delta_{\Gamma}$ are defined by $\left[11,(1.8)\right.$ and (1.9)] and $\operatorname{GL}\left(m,-q^{\delta}\right)=$ $\mathrm{U}\left(m, q^{\delta}\right)$ for all $\delta \geq 1$. Let $s^{*}$ be a dual of $s$ in $I_{0}(V)$ defined by [3, (4A)] with the primary decomposition $\prod_{\Gamma} s_{\Gamma}^{*}$. By $[3,(4.1)]$

$$
C_{I_{0}(V)}\left(s^{*}\right) \simeq C_{I_{0}(V)^{*}}(s)^{*}
$$

and by definition, $m_{\Gamma}\left(s^{*}\right)=m_{\Gamma}(s)$ for $\Gamma \neq X-1$ and $V_{X-1}$ is the underlying space of $s_{X-1}^{*}$, therefore

$$
C_{I(V)}\left(s^{*}\right) \simeq \prod_{\Gamma \neq X-1} \mathrm{GL}\left(m_{\Gamma}\left(s^{*}\right), \varepsilon_{\Gamma} q^{\delta_{\Gamma}}\right) \times I\left(V_{X-1}\right) .
$$

But the number of unipotent conjugacy classes of $\mathrm{GL}\left(m, \varepsilon q^{\delta}\right)$ for any sign $\varepsilon= \pm$ and $\delta \geq 1$ is the number of partitions of rank $m$, so $\mathscr{W}(B)$ is the number of unipotent conjugacy classes of $C_{I(V)}\left(s^{*}\right)$. Thus we have proved the following.

Proposition (1E). Let $B$ be a 2-block of $I(V)$ with a semisimple label $s^{*}$ for some semisimple $2^{\prime}$-element $s^{*}$ of $I_{0}(V)$. Then the number $\mathscr{W}(B)$ of $B$-weights is the number of unipotent conjugacy classes $\mathscr{U}_{I(V)}\left(s^{*}\right)$ of $C_{I(V)}\left(s^{*}\right)$. In particular, if $\prod_{\Gamma} s_{\Gamma}^{*}$ is the primary decomposition of $s^{*}$ in $I(V)$ in the sense of $[11, \mathrm{p}$. 125], then

$$
\mathscr{U}_{I(V)}\left(s^{*}\right)=\prod_{\Gamma} f_{\Gamma},
$$

where $f_{\Gamma}$ is the number of partitions of multiplicity $m_{\Gamma}\left(s^{*}\right)$ of the elementary divisor $\Gamma$ in $s^{*}$ except when $\Gamma=X-1$, in which case $f_{X-1}$ is given by (1.6) or (1.9) with $V$ replaced by the underlying space of $s_{X-1}^{*}$ according as $V$ is symplectic or orthogonal.

Remark (1F). As a corollary of (1E), we can get an affirmative answer for Alperin's weight conjecture for $I(V)$. Indeed, if $\mathscr{W}(I(V))$ is the number of weights of $I(V)$, then by $(1 \mathrm{E})$,

$$
\mathscr{W}(I(V))=\sum_{s^{*}} \mathscr{U}_{I(V)}\left(s^{*}\right),
$$

where $s^{*}$ runs over all semisimple $2^{\prime}$-elements of $I(V)$. Now the right-hand side of (1.11) is the number of conjugacy classes of 2-regular elements in $G$ and it is the number of irreducible Brauer characters $l(I(V))$ of $I(V)$ by a result of Brauer. Thus $\mathscr{W}(I(V))=l(I(V))$ and the remark follows.

\section{THE NUMBER OF IRREDUCIBLE BRAUER CHARACTERS}

The notation and terminology of $\S 1$ are continued in this section. The number of irreducible Brauer characters in a 2-block of a symplectic or special orthogo- 
nal group will be given and the weight conjecture of Alperin will be confirmed block by block for a symplectic or odd-dimensional orthogonal group.

The proof of the following proposition was pointed out by the referee of [3].

Proposition (2A). Let $q$ be a power of an odd prime, $G=I_{0}(V), B$ a 2-block of $G$, and $l(B)$ the number of irreducible Brauer characters in $B$. If $B=$ $\mathscr{E}_{2}(G,(s))$ for some semisimple $2^{\prime}$-element $s$ of the dual group $G^{*}=I_{0}(V)^{*}$, then $l(B)$ is the number of unipotent conjugacy classes of $C_{G^{*}}(s)^{*}$.

Proof. Let $t^{*}$ be a semisimple $2^{\prime}$-element of $G$, and let $t$ be its dual given by $[3,(4 \mathrm{~A})]$, so that $C_{G}(t) \simeq C_{G^{*}}(t)^{*}$. Let $\mathscr{U}(t)^{*}$ be the number of unipotent conjugacy classes of $C_{G}\left(t^{*}\right)$. If $\operatorname{dim} V \leq 2$, then it is trivial to check that $l(B)=\mathscr{U}\left(s^{*}\right)$. Suppose $s \neq 1$. Then $C_{G^{*}}(s)$ is a proper regular subgroup of $G^{*}$ and $C_{G}\left(s^{*}\right)$ is its dual group. By Broue [5, Theorem 2.3] there is a perfect isometry between $B$ and $\mathscr{E}_{2}\left(C_{G}\left(s^{*}\right),(1)\right)$. It follows that $l(B)$ is the number of irreducible Brauer characters of $\mathscr{E}_{2}\left(C_{G}\left(s^{*}\right),(1)\right)$. Let $\Pi_{\Gamma} s_{\Gamma}^{*}$ be the primary decomposition of $s^{*}$ in $G$ and $V_{X-1}$ the underlying space of $s_{X-1}^{*}$. Then

$$
C_{G}\left(s^{*}\right) \simeq\left(\prod_{\Gamma \neq X-1} \operatorname{GL}\left(m_{\Gamma}(s), \varepsilon_{\Gamma} q^{\delta_{\Gamma}}\right)\right) \times I_{0}\left(V_{X-1}\right)
$$

and $\operatorname{dim} V_{X-1}<\operatorname{dim} V$, so by induction $l\left(\mathscr{E}_{2}\left(I_{0}\left(V_{X-1}\right)\right),(1)\right)$ is the number of unipotent conjugacy classes of $I_{0}\left(V_{X-1}\right)$. By $[10, \S 8] l\left(\mathrm{GL}\left(m_{\Gamma}(s), e_{\Gamma} q^{\delta_{\Gamma}}\right)\right)$ is the number of partitions of rank $m_{\Gamma}(s)$. Thus $l(B)=\mathscr{U}\left(s^{*}\right)$. Now let $s=1$. The number of irreducible Brauer characters $l(G)$ of $G$ is

$$
l\left(B_{0}\right)+\sum_{t^{*} \neq 1} \mathscr{U}\left(t^{*}\right)
$$

where $t^{*}$ runs over all representatives for the semisimple conjugacy $2^{\prime}$-classes of $G$ with $t^{*} \neq 1$. By a result of Brauer $l(G)$ is the number of conjugacy $2^{\prime}$-classes in $G$, so that

$$
l(G)=\mathscr{U}(1)+\sum_{t^{*} \neq 1} \mathscr{U}\left(t^{*}\right)
$$

It follows that $l\left(B_{0}\right)=\mathscr{U}(1)$ and this proves $(2 \mathrm{~A})$.

Proposition (2B). Let $V$ be a symplectic or odd-dimensional orthogonal space and $B$ a 2-block of $I(V)$ such that $B$ covers a 2-block $B^{\prime}=\mathscr{C}_{2}\left(I_{0}(V),(s)\right.$ ) of $I_{0}(V)$, where $s$ is a semisimple $2^{\prime}$-element of the dual group $I_{0}(V)^{*}$ of $I_{0}(V)$. Let $s^{*}$ be a dual of $s$ in $I_{0}(V)$ given by [3, (4A)], and let $\mathscr{U}_{I(V)}\left(s^{*}\right)$ be the number of unipotent conjugacy classes of $C_{I(V)}\left(s^{*}\right)$. In addition, let $\mathscr{W}(B)$ be the number of $B$-weights. If $l(B)$ is the number of irreducible Brauer characters in $B$, then $l(B)=\mathscr{W}(B)=\mathscr{U}_{I(V)}\left(s^{*}\right)$. In particular, Alperin's weight conjecture holds for $B$ block by block.

Proof. Let $\mathscr{U}_{I_{0}(V)}\left(s^{*}\right)$ be the number of unipotent conjugacy classes of $C_{I_{0}(V)}\left(s^{*}\right)$. If $V$ is symplectic, then $I(V)=I_{0}(V)$ and $C_{I(V)}\left(s^{*}\right) \simeq C_{I(V)^{*}}(s)^{*}$. By $(2 \mathrm{~A})$ and $(1 \mathrm{E}) l(B)=\mathscr{U}_{I(V)}\left(s^{*}\right)=\mathscr{W}(B)$. If $V$ is odd-dimensional orthogonal, then $I(V)=\left\langle-1_{V}\right\rangle \times G$ and $C_{I(V)}\left(s^{*}\right)=\left\langle-1_{V}\right\rangle \times C_{I_{0}(V)}\left(s^{*}\right)$, where $1_{V}$ is the identity of $I(V)$. Thus $\mathscr{U}_{I(V)}\left(s^{*}\right)=\mathscr{U}_{I_{0}(V)}\left(s^{*}\right)$ and $l(B)=l\left(B^{\prime}\right)$, so that (2B) follows from $(2 \mathrm{~A})$ and $(1 \mathrm{E})$. 
Remark (2C). In the notation of (2B), suppose $V$ is even-dimensional orthogonal. If the multiplicity $m_{X-1}\left(s^{*}\right)$ of $X-1$ in $s^{*}$ is zero, then $\mathscr{U}_{I(V)}\left(s^{*}\right)=l(B)$ and so Alperin's weight conjecture has an affirmative answer for $B$. Indeed, in this case $C_{I(V)}\left(s^{*}\right)=C_{I_{0}(V)}\left(s^{*}\right)$, so that $\mathscr{U}_{I(V)}\left(s^{*}\right)=\mathscr{U}_{I_{0}(V)}\left(s^{*}\right)=l\left(B^{\prime}\right)$. Let $\tau$ be an involution of $I\left(V^{*}\right)$ with determinant -1 , where $V^{*}$ is the underlying space of $I_{0}(V)^{*}$. Then $\mathscr{E}_{2}\left(I_{0}(V),\left(s^{\tau}\right)\right)$ is a block $B^{\prime \prime}$ of $I_{0}(V)$ and $B$ covers $B^{\prime \prime}$ by [3, (5B)]. Since $s$ is not conjugate with $s^{\tau}$ in $I_{0}(V)^{*}$, it follows that $B^{\prime} \neq B^{\prime \prime}$, so that $B^{\prime \tau}=B^{\prime \prime}$ and $\tau \notin N\left(B^{\prime}\right)$, where $N\left(B^{\prime}\right)$ is the stabilizer of $B^{\prime}$ in $I(V)$. Thus $N\left(B^{\prime}\right)=I_{0}(V)$ and $l(B)=l\left(B^{\prime}\right)$ by a result of Fong and Reynolds [9, Theorem V.2.5]. It follows that $\mathscr{U}_{I(V)}\left(s^{*}\right)=l(B)$ and the remark follows.

\section{REFERENCES}

1. An Jianbei, 2-weights for general linear groups, J. Algebra 149 (1992), 500-527.

2. __ 2-weights for unitary groups, Trans. Amer. Math. Soc. 339 (1993), 251-278.

3. _ 2-weights for classical groups, J. Reine Angew. Math. 439 (1993), 159-204.

4. M. Broué, Les l-blocs des groupes $\mathrm{GL}(n, q)$ et $\mathrm{U}\left(n, q^{2}\right)$ et leurs structures locales, Sémainaire Bourbaki Astérisque 640 (1986), 159-188.

5. $\longrightarrow$, Isométries parfaites, types de blocs, catégories dérivées, Astérisque 181-182 (1990), 61-92.

6. M. Broué and J. Michel, Blocs et séries de Lusztig dans un groupe réductif fini, I. Reine Angew. Math. 395 (1989), 56-67.

7. M. Cabanes and M. Enguehard, Unipotent blocks of finite reductive groups with a given type, Math. Z. 213 (1993), 479-490.

8. R. Carter, Finite groups of Lie type: conjugacy classes and complex characters, Wiley/ Interscience, New York, 1985.

9. W. Feit, The representation theory of finite groups, North-Holland, Amsterdam, 1982.

10. P. Fong and B. Srinivasan, The blocks of finite general linear and unitary groups, Invent. Math. 69 (1982), 109-153.

11.

12. J. Olsson, McKay number and height of characters, Math. Scand. 38 (1976), 25-42.

13. _ Remarks on symbols, hooks, and degrees of unipotent characters, J. Combin. Theory Ser. A 42 (1986), 223-238.

14. G. E. Wall, On the conjugacy classes in the unitary, symplectic and orthogonal groups, J. Austral. Math. Soc. 3 (1963), 1-26.

Department of Mathematics and Statistics, University of Auckland, Private Bag 92019, AuCKLAND, New Zealand

E-mail address: an@mat .auckland.ac.nz

E-mail address: conder@mat.auckland.ac.nz 